\title{
Subject Index 1993
}

Acute leukemia Suppl. 120

Acute promyelocytic leukemia 122

Adjuvant chemotherapy 3 158, Suppl. 126

Adjuvant immunotherapy 3158

Adjuvant irradiation 6416

Adjuvant therapy 5 297, 304, 6 416, Suppl. 1 23, 26, 32

Alkylglycerols 3170

Alkylphosphocholine 3 170, 4260

Alkylphospholipids Suppl. 17

Aminogluthetimide Suppl. 16

Anthracycline Suppl. 18

Antiestrogens Suppl. 15

Antimicrotubule agent 5329

Antineoplastic activity 5329

Antitumor lipids 3 170, 4260

ARA-C

- low dose Suppl. 121

«Arbeitsgruppe Wirkstoifentwicklung» (AWO) Suppl. 15

Aromatase inhibitors Suppl. 16

Autochthonous tumors Suppl. 15

AWO Suppl. 15

Colorectal tumor 4232

Conformal radiotherapy 6389

Conventional-dose chemotherapy 5310

Coping 5371

Coping with disease 3178

Costimulation 5290

19

Cyclosporin A/Ciclosporin A 1 26, Suppl.

Cystoprostatectomy Suppl. 126

Cytostatic effects 126

2'-Deoxycoformicin Suppl. 119

Dexniguldipine Suppl. 125

Dexverapamil Suppl. 112

Differentiated follicular thyroid carcinoma 4274

Doppler 3183

Dose escalation 3 174, 5 310, Suppl. 120

Dose intensity 5344

Doxorubicin Suppl. 19, 25 
- pharmacokinetics 116

Drug resistance 4264

Durametastasis 4274

Biopsy 5354

Bladder carcinoma 3 158, Suppl. 126

- $\quad$ localized invasive Suppl. 126

Bone marrow transplantation 137,5310 Brachytherapy in prostate cancer 3150 Breast cancer 3

170, 4 260, 5 297, 304, 329, 364, 6 434, 450, Suppl. 1 7, 29

- $\quad$ prevention 5297

Breast carcinoma 3183

Breast-preserving therapy 5364

Breast reconstruction 5364

Breast vascularity 3183

Budotitane Suppl. 111

CA72-4 3199

CA125 3199

Cancer cachexia Suppl. 116

Cancer information service 5371

Carboplatin

- $\quad$ intraperitoneal 5360

Carcinoma of the penis 294

Cardiotoxicity 4245

Catalase 3189

Chemotherapy 1 32, 2 88, 3 183, 4 252, 6407

high dose Suppl. 129

preoperative Suppl. 130,31 2-chlorodeoxyadenosine Suppl. 119

Chronic lymphocytic leukemia (CLL) Suppl. 1 13, 19

Ciclosporin A/Cyclosporin A 1 26, Suppl. 19

Cisplatin 4270

Clinical trial 111

Colon and rectum carcinoma 6416

Colon cancer Suppl. 132

Colorectal cancer Ill,4 222, Suppl. 132

Colorectal carcinoma 4 252, 5 290, Suppl. 132

Early diagnosis 261 Early gastric cancer 5354 Early recurrance 6434 Elderly patients 6440

Endoscopic sonography 6425 Endothelial cell 4270 Enteral diet and cancer Suppl. 116 ENT

tumors 3195 Epirubicin Suppl. 19 Erythropoietin 279 Erythropoietin therapy Suppl. 121

Esophageal carcinoma Suppl. 130 Ether lipids 111 Etoposide 6440

Fludarabine phosphate Suppl. 113

5-Fluorouracil 4 245, 252, 6 416, Suppl. 123

5-Fluorouracil/FUDR Suppl. 123

Fluorouracil 4232

Folinicacid 4 245,252,6 416

Follow-up 6425

FOM chemotherapy 2100 
Fotemustine Suppl. 115

Gastric cancer Suppl. 131 - locally advanced Suppl. 131 Gastroscopy 5354 G-CSF 5 310, Suppl. 120 Gemcitabine Suppl. 115 Germ cell tumor 288 Glioblastoma 122 Glutathione Stransferase 3189 GM-CSF 5 310, Suppl. 1 20, 21 Growth factor Bi Suppl. 128

Hairy cell leukemia Suppl. 119

Heart transplantation 5338

Hematopoietic growth factors Suppl. 120

Hemoglobin 279

Heparin 116

Hepatitis B 132

Hexadecylphosphocholine 1 11, 3 170, 4 260, Suppl. 1 7, 8

HIV-associated lymphomas Suppl. 118

Hodgkin's disease 6407

Hodgkin's lymphoma Suppl. 118

Hormonally active substances Suppl. 15

Hormone receptor superfamily Suppl. 128

Human tumor xenografts Suppl. 15

Hypernephroma Suppl. 125

Imaging 3150

Immune parameters Suppl. 116

Immune therapy Suppl. 129

Immunocytochemistry 4264

Immunological findings Suppl. 118

Immunosuppression 5 338, Suppl. 128

Immunotherapy Suppl. 124

Information transfer 5371

Inguinal lymphadenectomy 270

Integrin expression Suppl. 17

Interferon 132

Interferon $\alpha$ Suppl. 1 7, 19, 23, 24, 29

Interferon $\alpha 2 \mathrm{a}$ Suppl. 123

Interferon $\alpha 2 b$ Suppl. 1 15, 25

Interferon $\beta$ Suppl. 114

Interferon $\gamma 6461$

Interleukin 2 Suppl. 1 24, 25, 29

Interleukin 3 Suppl. 121

Intraoperative sonography 6425

Intratumoral application Suppl. 114

Intravesical therapy 3158

In vitro short-term test 3189

Iododoxorubicin 6446

Isoelastic shoulder prosthesis 2106

Isolated perfusion 116

Laser surgery 270 Leukemia

acute 4264

lymphoblastic 4264 
nonlymphoblastic 4264 Levamisole 6416 Liaison-consultation services 137 Lobaplatin Suppl. 1 13, 14 Long-term survival 15

Lung cancer

small cell Suppl. 128 Lung carcinoma 3189 Lymphadenectomy

retroperitoneal Suppl. 126 Lymph node negative breast cancer

vascular invasion 15

Malignancy 5338 Malignant lymphoma 3195 Melanoma 116, 3163

malignant 4 222, Suppl. 115 , 29,30

oncolysates, viral 5290

Melphalan 3163

Metallothionein 3189

Metastatic breast cancer 5344

5,10 Methylenetetrahydrofolate Suppl. 132

Miltefosine Ill,3 170, 4260

Mini-port 6454

Mitotic rate 15

Mitoxantrone 6440

Molecular biological findings Suppl. 118

Monitoring therapy 3150

Multidrug resistance 2 114, 6 450, Suppl. 1 8, 12

Multimodality therapy Suppl. 130

M-VAC Suppl. 126

Myelodysplasia Suppl. 121

Myelodysplastic syndromes Suppl. 1 20, 21

Myelodysplastic syndromes (FAB-Type I and II) Suppl. 121

Myocardial infarction 288

N-Benzyladriamycin-14-valerate Suppl. 18

ND:YAG laser coagulation 294

Need for information 5371

Neo-adjuvant therapy Suppl. 126

Neoplasm 4232

Nephrectomy Suppl. 124

Neutropenia 5310

Newcastle disease virus (NDV) 5290

NK61 1 Suppl. 1 11, 12

Non-cisplatin-based chemotherapy 2100

Non-Hodgkin's lymphoma 1 32, 6 440, Suppl. 119

low grade Suppl. 119

high grade Suppl. 120 Non-seminomatous germ cell tumors Suppl. 126 Non-small-cell lung cancer (NSCLC) 2 100, 5 329, 6 446,

Suppl. 1 13,28 Nucleoside-analogs Suppl. 119 Nutritional therapy Suppl. 116 parenteral Suppl. 117

Nutrition and cancer Suppl. 116

Octreotide Suppl. 122 Organ-preserving therapy 294 Osteolytic bone disease 3174 Ovarian cancer 4 222, 5329 Ovarian cancer cell lines 2114 Ovarian carcinoma 3199 
- $\quad$ bulky disease 5360

Oxazaphosphorines Suppl. 16

Oxygenation 279

Pain 3174

Palliation 2 100, 3174

Palliative care 2 Ill

Pamidronate 3174

Pancreatic cancer Suppl. 115

PAS port 6454

Penile carcinoma 270

Pentostatin Suppl. 119

Perhydrothiazinylphosphamide ester Suppl. 16

PET 4232

P-glycoprotein 3 189, 4 264, Suppl. 18

P-glycoprotein 1702114

Phase II 111

Phase II study 1 29, 3 170, 4 260, 6446

Pirarubicin 6450

Podophyllotoxin Suppl. 1 11, 12 Port systems 6454 Pouches Suppl. 126

Precancerous lesions 5354 Prednisolone 6440 Prevention trial 5297 Prognosis 6434 Prognostic parameters 3158 Prognostic subgroups 5344 Prostate 6389 Prostate cancer 3150 Prostate carcinoma 3195 Psychooncology 3178 Psychosomatics 137 Pulmonary embolism 288

Quality of life 2100,5344 Quinidine 6450

Radiation therapy 3195

Radiobiology 279

Radio-/Chemotherapy

- praeoperative Suppl. 128,30

Radioimmunodetection 4222

Radioimmunoimaging 6465

Radiooncology 3178

Radiotherapy 6389,407

Rats 4270

Raynaud's phenomenon 288

Rectal cancer Suppl. 132

Refractory malignant ascites 2 Ill

Regional isolated perfusion 3163

Rehabilitation of carcinoma patients 141

Relapse therapy 6407

Renal cell cancer 6 461, Suppl. 1 23, 24, 25

Renal cell carcinoma Suppl. 19, 12, 24, 25

Resistance mechanisms 3189

Reticulum cell sarcoma 126

Retinoic acid metabolism 122

Risk factors 5297

Ruthenium complexes Suppl. 16

Sarcoma 116 
Serum thymidine kinase 3195 Small cell lung cancer Suppl. 114 Social worker 141

Somatostatin analog Suppl. 122 Squamous cell carcinoma 6465 SRA 3199

Staging 6425,465

Steroid synthesis modulators 122

Stroke 288

Subcutaneous mastectomy 5364

Subrenal capsule assay 126

Survival 5344

Survival rates 2106

Tamoxifen 5297

high dose Suppl. 17 Target volume 6389 Taxotere Suppl. 113 T-cell activatin 5290 Testicular cancer 1 29, Suppl. 126

chemotherapy Suppl. 127

salvage therapy Suppl. 127 Testicular intraepithelial neoplasia 261 Testicular neoplasms 261 Test systems Suppl. 15 Thermochemotherapy Suppl. 18 Thymidylate synthase 3189 Thyroid cancer Suppl. 122

- $\quad$ chemotherapy Suppl. 122,23

Thyroid carcinoma Suppl. 1 22, 23

Titanocene dichloride Suppl. 1 9, 10

Topoisomerase II 3189

Toxicity 2100

Transfusion 279

Transitional cell cancer Suppl. 126

Transrectal ultrasonography 3150

Transrectal ultrasound 6389

Tubular formation 15

Tumor antibodies 4222

Tumor cells

- $\quad$ circulating Suppl. 130

Tumor-cell vaccines 5290

Tumor marker 3199

Tumor necrosis factor 2 Ill

Tumors in the proximal humerus 2106 Tumor vascularity 3183 Tumour cachexia Suppl. 116

Tumour diagnosis 6425

Vaccination

- postoperative 5290

Vascular damage 4270

Vessel invasion 6434

Vinblastine Suppl. 123

Vinorelbine 129

484 